\title{
Splenic involvement in umbilical cord matrix-derived mesenchymal stromal cell- mediated effects following traumatic spinal cord injury
}

Anna Badner ${ }^{1,2}$, Justin Hacker ${ }^{1}$, James Hong ${ }^{1,2}$, Mirriam Mikhail ${ }^{1}$, Reaz Vawda ${ }^{1}$ and Michael G. Fehlings ${ }^{1,2,3^{*}}$ (D)

\begin{abstract}
Background: The spleen plays an important role in erythrocyte turnover, adaptive immunity, antibody production, and the mobilization of monocytes/macrophages $(\mathrm{M} \varphi)$ following tissue injury. In response to trauma, the spleen initiates production of inflammatory cytokines, which in turn recruit immune cells to the inflamed tissue, exacerbating damage. Our previous work has shown that intravenous mesenchymal stromal cell (MSC) infusion has potent immunomodulatory effects following spinal cord injury $(\mathrm{SCl})$, associated with the transplanted cells homing to and persisting within the spleen. Therefore, this work aimed to characterize the relationship between the splenic inflammatory response and SCl pathophysiology, emphasizing splenic involvement in MSC-mediated effects.
\end{abstract}

Methods: Using a rodent model of cervical clip-compression $\mathrm{SCl}$, secondary tissue damage and functional recovery were compared between splenectomised rodents and those with a sham procedure. Subsequently, 2.5 million MSCs from the term human umbilical cord matrix cells (HUCMCs) were infused via tail vein at 1-h post-SCl and the effects were assessed in the presence or absence of a spleen.

Results: Splenectomy alone had no effect on lesion volume, hemorrhage, or inflammation. There was also no significant difference between the groups in functional recovery and those in lesion morphometry. Yet, while the infusion of HUCMCs reduced spinal cord hemorrhage and increased systemic levels of IL-10 in the presence of a spleen, these effects were lost with splenectomy. Further, HUCMC infusion was shown to alter the expression levels of splenic cytokines, suggesting that the spleen is an important target and site of MSC effects.

Conclusions: Our results provide a link between MSC function and splenic inflammation, a finding that can help tailor the cells/transplantation approach to enhance therapeutic efficacy.

Keywords: Spleen, Spinal cord injury, Mesenchymal stromal cells

\section{Background}

Even with recent strides in patient care, spinal cord injury (SCI) remains a devastating and debilitating condition, where motor and sensory impairments hamper patient quality of life and present a major economic burden. While there is a significant need for better treatment options, few therapies succeed in clinical translation [1]. For

\footnotetext{
* Correspondence: Michael.Fehlings@uhn.ca

'Division of Genetics and Development, Krembil Research Institute, University Health Network, 60 Leonard Ave, Toronto, Ontario M5T 2S8, Canada ${ }^{2}$ Institute of Medical Science, University of Toronto, 1 King's College Circle, Toronto, Ontario M5S 1A8, Canada

Full list of author information is available at the end of the article
}

this reason, greater understanding of injury pathophysiology, especially in relation to treatment application, will be imperative for progress to occur.

Traumatic SCI, initiated by mechanical insult known as the primary injury, involves a secondary cascade of further damage. Specifically, the disruption of local vasculature results in hemorrhage, tissue ischemia, and permeability of the blood spinal cord barrier, thereby allowing for the infiltration of peripheral immune cells and release of inflammatory cytokines [2]. Through a positive feedback loop, chemoattractants and activated immune cells further contribute to a pro-inflammatory

(c) The Author(s). 2018 Open Access This article is distributed under the terms of the Creative Commons Attribution 4.0 International License (http://creativecommons.org/licenses/by/4.0/), which permits unrestricted use, distribution, and 
state, which exacerbates cell death and increases tissue damage. Yet, beyond localized spinal cord changes, increasing attention has been placed on the contribution of peripheral immune organs in SCI pathophysiology [3], with considerable focus on the spleen.

Briefly, the spleen plays an important role in erythrocyte turnover, adaptive immunity, antibody production, and, more recently revealed, the mobilization of monocytes/macrophages $(\mathrm{M} \phi)$ following tissue injury [4]. In the context of high-level SCI, several studies have evaluated dysfunctional antibody synthesis following loss of splenic sympathetic preganglionic innervation [5-7] and the production of autoantibodies against spinal cord tissue [8]. Yet, equally important is the acute response to trauma-induced inflammation, where the spleen initiates production of tumor necrotic factor- $\alpha$ (TNF $\alpha)$, mostly by resident $M \phi s$, and in turn influences the recruitment of immune cells to the inflamed tissue [9]. Relatedly, acute splenic atrophy and changes in the activation of residing $M \phi s$ as well as $T$ cells have been reported in models of acute brain ischemia [10-12], as well as SCI [13]. Further, targeting this peripheral inflammatory response, via splenectomy, has been shown to significantly reduce the number of infiltrating blood-derived $M \phi s$, thereby improving functional recovery following thoracic SCI [14]. In contrast, despite promising effects, splenectomy studies in brain injury have had mixed results $[15,16]$.

Notably, the application of mesenchymal stromal cells (MSCs) has further highlighted a link between the spleen and central nervous system injury. Intravenously infused MSCs have been shown to localize in the spleen following stroke $[17,18]$, traumatic brain injury $[19,20]$, and SCI $[21,22]$. Although this distribution may vary with source and recipient age [23], these studies have reported dramatic changes in splenic gene expression [21], cytokine production $[18,22]$, and the proportion of $\mathrm{CD} 4+\mathrm{T}$ cells [20] following MSC infusion. Therefore, expanding on these results, this study aimed to characterize the acute splenic cytokine response to SCI and determine its role in MSC-mediated recovery. Here, we demonstrate that cervical SCI induces a unique splenic inflammatory response, with significantly greater expression of TNF $\alpha$, as early as $1 \mathrm{~h}$ following trauma, which is followed by a pronounced influx of other pro-inflammatory cytokines, including IL-17, IL-13, IL-1 $\beta$, and CXCL10. Despite these implications, there was no difference detected in secondary tissue damage or long-term functional recovery when compared between splenectomised rodents and those with a sham procedure. However, while the infusion of term human umbilical cord matrix (HUCMCs) reduced spinal cord hemorrhage and increased systemic levels of interleukin-10 (IL-10) in the presence of a spleen, these effects were lost with splenectomy. Further, the HUCMCs were also shown to alter splenic cytokine expression levels following SCI, suggesting that the spleen is an important target and site of MSC effects (when systemically infused). Taken together, these results highlight splenic involvement in acute pathophysiology and demonstrate that peripheral immune tissue may be a minimally invasive therapeutic target for SCI, with important clinical implications.

\section{Methods \\ Clip-compression spinal cord injury (SCl)}

All animal experiments were approved by the animal care committee at the University Health Network (Toronto, Ontario, Canada) in compliance with the Canadian Council on Animal Care. Adult female (approximately $250 \mathrm{~g}$; 10-11 weeks old) Wistar rats (Charles River Laboratories, Wilmington, MA) were given buprenorphine $(0.05 \mathrm{mg} /$ $\mathrm{kg}$ ) and $5 \mathrm{~mL}$ of saline prior to surgery. All rats were anesthetized with isoflurane (delivered in a 1:1 mixture of $\mathrm{O}_{2} /$ $\mathrm{N}_{2} \mathrm{O}$ ) and received a C7-T1 laminectomy. Next, a 35-g aneurysm clip was applied to the C7 level (1 min), modeling traumatic cervical SCI. Buprenorphine, amoxicillin trihydrate/clavulanate potassium, and subcutaneous saline injections $(5 \mathrm{~mL})$ were administered postoperatively. Animals were housed at $26{ }^{\circ} \mathrm{C}$ in a 12-h light/dark cycle, and their bladders were manually expressed twice a day.

\section{Cytokine array for splenic response to $\mathrm{SCl} \& \mathrm{MSC}$ infusion} The spleens of naive, laminectomy-only, and SCI animals were collected at $1 \mathrm{~h}$ as well as $24 \mathrm{~h}$ following surgery. The tissue was snap-frozen on dry ice after transcardial perfusion (with $250 \mathrm{ml}$ of phosphate buffer solution; PBS) and stored for later use. When all samples were collected, the spleens were homogenized using radioimmunoprecipitation assay (RIPA) buffer (20-188, EMD Millipore) with proteinase inhibitors (Thermo Fisher Scientific), according to the manufacturer's instructions. Total protein levels were determined using a BCA Protein Assay Kit (Thermo Fisher Scientific). The homogenate was diluted, and $100 \mu \mathrm{g}$ of protein assessed with the cytokine R\&D ELISA Proteome Profiler array (ARY008, R\&D Systems Inc.) as per manufacturer's instructions. To increase detection sensitivity, IRDye 800CW Streptavidin (926-32230, LI-COR) was used at a 1:2000 dilution (30 min, room temperature) as replacement for the kit's streptavidin-horseradish peroxidase. The array membranes were scanned on an Odyssey Imager CLx (LI-COR), and images quantified as previously described with a semi-automated ImageJ macro [22]. The same approach was applied for the splenic cytokine profile following MSC infusion.

\section{Splenectomy}

All Wistar rats received amoxicillin trihydrate/clavulanate potassium in water prior to the procedure. In a clean surgical space and under isoflurane anesthesia, the abdomen was accessed through a midline laparotomy. The spleen was pulled through the incision, and splenic 
vessels ligated prior to removal. In sham splenectomies, the spleen was returned to the abdominal cavity and the incision was closed via muscle and skin layers. Buprenorphine $(0.05 \mathrm{mg} / \mathrm{kg})$ and $5 \mathrm{~mL}$ of saline were administered postoperatively.

\section{In vivo very high-resolution ultrasound and Power Doppler imaging}

In vivo very high-resolution ultrasound (VHRUS) and Power Doppler imaging was performed as previously described [24]. Briefly, a customized stabilization frame was used to fix the animal position on an imaging platform (Vevo Imaging Station, VisualSonics, Toronto, Ontario). The injury was exposed and ultrasound gel (scanning gel, Medi-Inn, Cameron, Ontario) placed on the dura mater. The VHRUS probe (44 MHz, Vevo 770, VisualSonics, Toronto, Ontario) was used to scan the spinal cord in three-dimensional (3D) B-Mode. The acute lesion and 8-week cavity volume were assessed with the TrakEM2 plugin on ImageJ software [22].

\section{Drabkin's assay for intraparenchymal hemorrhage}

As previously described [25], intraparenchymal hemorrhage post-SCI was assessed with Drabkin's assay. In short, a 5-mm segment of the injured spinal cord was collected after transcardial perfusion with $250 \mathrm{~mL}$ of PBS. The samples were subsequently homogenized in $100 \mu \mathrm{l}$ of distilled water and spun down at $13000 \mathrm{rpm}$ for $15 \mathrm{~min}$. The supernatant was collected and $20 \mu \mathrm{l}$ added to $80 \mu \mathrm{l}$ of Drabkin's reagent (Drabkin's Reagent powder [D5941] Sigma-Aldrich, in $1000 \mathrm{ml}$ of distilled $\mathrm{H}_{2} \mathrm{O}$ and $0.5 \mathrm{ml}$ of $30 \%$ Brij 35 Solution) in a clear 96-well plate. The reaction was incubated for $15 \mathrm{~min}$ and absorbance read at $560 \mathrm{~nm}$ (Wallac 1420 VICTOR2; PerkinElmer).

\section{Long-term neurobehavioral assessment}

All neurobehavioral assessments were performed weekly (for 8 weeks) following SCI by two independent observers blinded to the experimental groups $(n=10$ per group). As previously described, forelimb motor function was measured with a grip strength meter (SDI Grip Strength System, model DFM-10; San Diego Instruments, San Diego, CA) [26], trunk stability was evaluated with the inclined plane test [27], and hind limb locomotion was assessed by using the 22-point (0-21) Basso, Beattie, and Bresnahan (BBB) locomotor rating scale [28]. When animals reached consistent weight bearing ability (week 5-6), the CatWalk multivariate system (Noldus, Version 7.1) was used to determine detailed parameters of paw and limb function during locomotion. No food restriction or reward was used to motivate the animals to walk, and successful performance required three complete crossings with at least three consecutive step cycles for analysis. Prints were labeled by an observer blinded to the experimental groups (at week 6 and 8 post-SCI).

\section{Lesion morphometry}

Under isoflurane anesthesia, animals were transcardially perfused with $180 \mathrm{~mL}$ of PBS and another $60 \mathrm{~mL}$ of chilled $4 \%$ paraformaldehyde (PFA). A 1-cm-length spinal cord segment (spanning the lesion epicenter) was isolated, postfixed for 1 day, and then cryoprotected in 30\% sucrose before embedding in OCT medium (Thermo Fisher Scientific, Oakwood Village, $\mathrm{OH}$ ). The spinal cord was cut into $30-\mu \mathrm{m}$ transverse sections and stained with luxol fast blue (LFB) and hematoxylin-eosin (H\&E) as previously described [29]. Lesional tissue, gray matter, and white matter percent area (sampled at every $240 \mu \mathrm{m}$ over a distance of $2400 \mu \mathrm{m}$ ) were quantified by an observer blinded to the experimental groups using the Cavalieri probe on Stereo Investigator (MBF Bioscience, Williston, VT).

\section{Cell isolation, culture, immunophenotypic antigen profiling, and intravenous delivery}

Umbilical cord tissue was obtained postpartum (Chelsea and Westminster Hospital, London, UK), and the veins/arteries removed under aseptic conditions. Subsequently, the Wharton's jelly (also known as umbilical cord matrix) was diced, incubated with collagenases I and II $(1 \mathrm{mg} / \mathrm{ml}$, Thermo Fisher Scientific, Oakwood Village, $\mathrm{OH}$ ) for $2 \mathrm{~h}$ (at $37{ }^{\circ} \mathrm{C}$ ), and centrifuged at $2000 \mathrm{rpm}$ for $10 \mathrm{~min}$. The obtained human umbilical cord matrix cells (HUCMCs) were plated on culture T175 flasks (Greiner Bio-One, Monroe, $\mathrm{NC})$ in minimum essential medium Eagle $\alpha$ modification $(\alpha-\mathrm{MEM})$ with $10 \%$ fetal bovine serum (FBS) (Wisent Bioproducts, St. Bruno, Quebec) and 0.1\% gentamycin (Sigma-Aldrich, St. Louis, MO). HUCMCs were grown to $80 \%$ confluence and used at passages 7-8. Prior to in vivo application, immunophenotypic cell antigen profiling was completed with a mesenchymal cell characterization kit (1:500; SCR067, EMD Millipore, Billerica, MA) in addition to mouse anti-human CD13 (1:500; C8589, Sigma-Aldrich) and 4',6-diamidino-2-phenylindole (DAPI; 1:1000) as nuclear counterstain. HUCMCs expressed mesenchymal cell markers sCD73, CD146, CD44, CD13, and PDGFR (Fig. 5b). For in vivo experiments, 2.5 million passage-matched (7-8) HUCMCs in $1 \mathrm{ml}$ of Hanks' buffer/2 mM EDTA (HE) were slowly infused $(200 \mu \mathrm{l} /$ minute $)$ via the tail vein at $1 \mathrm{~h}$ following SCI.

\section{Interleukin-10 (IL-10) enzyme-linked immunosorbent assay (plasma)}

Plasma levels of IL-10 were evaluated at $24 \mathrm{~h}$ following SCI and in SCI with HUCMC infusion. Blood samples were collected via cardiac puncture, prior to transcardial perfusion, into EDTA-coated Vacutainer tubes (K2 EDTA Plus Blood Collection Tubes, BD Biosciences, San Jose, CA). The 
samples were subsequently centrifuged at $3000 \mathrm{rpm}$ (centrifuge $5810 \mathrm{R}$, Eppendorf) for $10 \mathrm{~min}\left(4^{\circ} \mathrm{C}\right)$, and the plasma was collected. The plasma samples were applied for the rat interleukin-10 (IL-10) enzyme-linked immunosorbent assay (ELISA) kit (AB100765, Abcam) per the manufacturer's instructions, and the absorbance was read at $450 \mathrm{~nm}$ (Wallac 1420 VICTOR2; PerkinElmer, Waltham, MA).

\section{Statistical analysis}

Quantitative data are expressed as the mean \pm standard error of the mean (SEM). The R\&D ELISA Proteome Profiler array results were normalized to laminectomy-only surgeries assessed by multiple $t$ tests with Holm-Sidak correction for multiple comparisons. Other differences between groups were assessed by unpaired $t$ test as well as two-way analysis of variance (ANOVA) with Tukey's post hoc tests (statistically significant at $p \leq 0.05$ ). All data were analyzed with GraphPad Prism (GraphPad Software Inc., La Jolla, CA, USA, http://www.graphpad.com).

\section{Results}

The spleen is a site of a $\mathrm{SCl}$-induced inflammation

Splenic cytokine changes were evaluated with the $R \& D$ ELISA Proteome Profiler array (Fig. 1). Within the first hour following SCI, a significant $(p=0.002)$ rise in splenic TNF $\alpha$ levels was detected when compared to laminectomy-only surgery animals $(n=4$ per group, multiple $t$ tests, Holm-Sidak correction for multiple comparisons). This was followed by a pronounced influx in the expression of IL-17 $(p=0.04), \mathrm{IL}-13(p=0.02), \mathrm{IL}-1 \beta(p=0.03), \operatorname{CXCL10}(p=$ $0.002)$, and $\operatorname{sICAM}(p=0.003)$ at 24 -h post-SCI. Similarly, although the levels of IL- 6 and thymus chemokine/CXCL7 were relatively lower than those in time-matched naive animals, expression also increased $24 \mathrm{~h}$ following injury. There

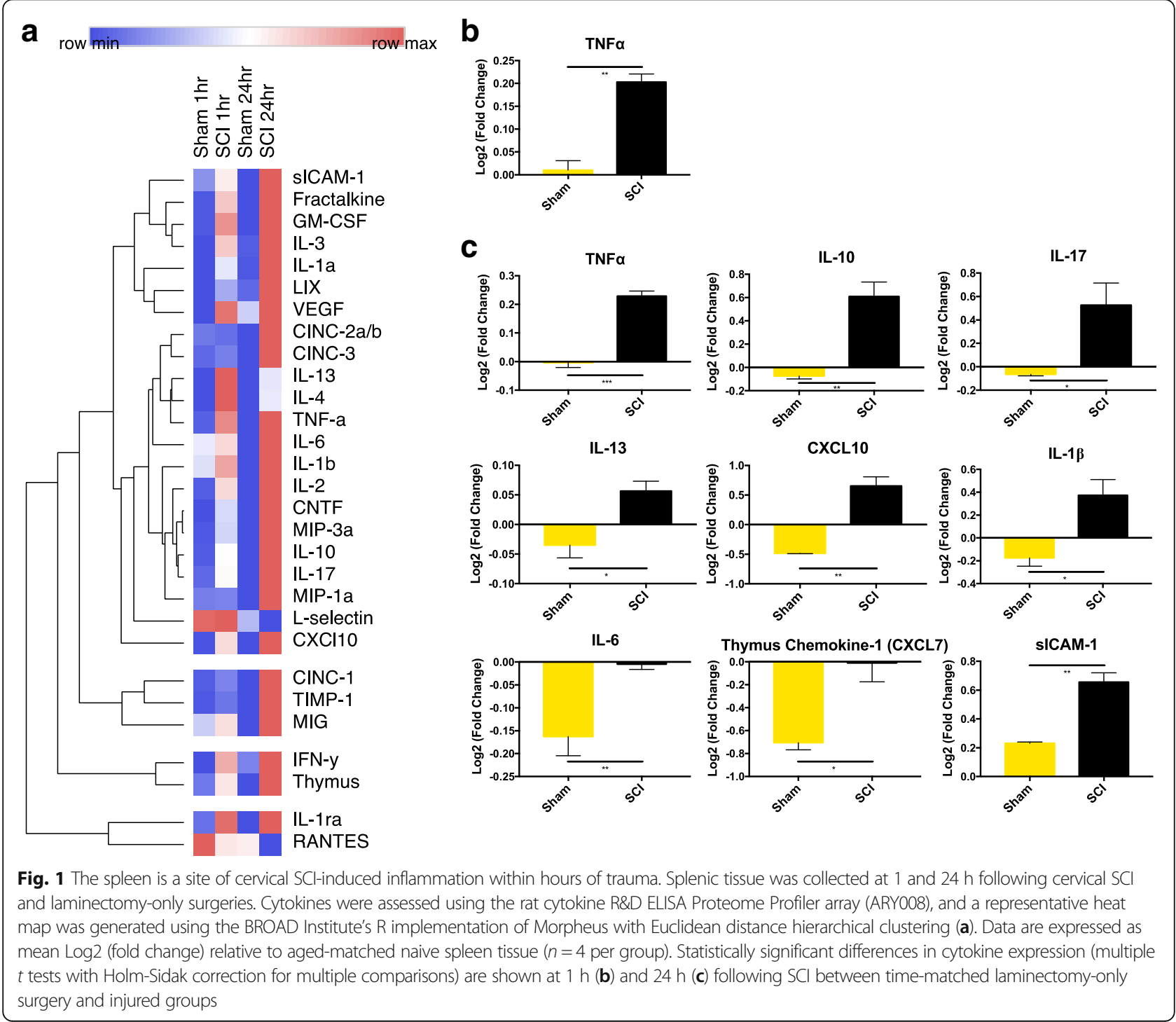


was no significant difference in spleen weight or length (Additional file 1: Figure S1).

\section{Splenectomy does not reduce parenchymal hemorrhage and lesion volume following $\mathrm{SCl}$}

In order to understand how the spleen contributes to secondary pathology, parenchymal hemorrhage and lesion volume were compared between splenectomised rodents versus those with a sham procedure (Fig. 2). Although SCI prompted splenic inflammation, there was no significant difference in spinal cord blood volume at $1(p=0.71), 3(p$ $=0.25)$, and $7(p=0.36)$ days post-injury (unpaired $t$ tests). Similarly, the lesion volume, quantified via in vivo VHRUS imaging, was also unaffected by splenectomy at $1(p=0.34)$, $3(p=0.82)$, and $7(p=0.07)$ days post-SCI.

\section{Splenectomy does not result in long-term functional} recovery and tissue preservation following $\mathrm{SCl}$

Long-term functional recovery ( $n=10$ per group) was assessed with a battery of standardized neurobehavioral tests (Fig. 3). Consistent with the acute study, splenectomy did not lead to changes in grip strength $(p=0.07$, two-way ANOVA), the inclined plane test $(p=0.92$, two-way ANOVA), or the BBB locomotor score $(p=0.79$, two-way ANOVA) as well as sub-score ( $p=0.62$, two-way ANOVA). Animal gait parameters were also evaluated with the CatWalk system at 6 and 8 weeks post-SCI (Fig. 3c, d). No significant differences were detected in forelimb/hind limb print area $(p=0.67$ for forelimbs and $p=0.85$ for hind limbs, two-way ANOVA), swing speed ( $p=0.56$ for forelimbs and $p=0.18$ for hind limbs, two-way ANOVA), stride length $(p=0.28$ for forelimbs and $p=0.07$ for hind limbs, two-way ANOVA), and stand time ( $p=0.78$ for forelimbs and $p=0.93$ for hind limbs, two-way ANOVA).

The absence of changes at 8 weeks with splenectomy was further confirmed with histomorphometric analysis $(n=4$ per group, two-way ANOVA) of LFB- and H\&E-stained sections (Fig. 4). There was no effect on lesional tissue ( $p=$ $0.11)$, gray $(p=0.44)$, or white $(p=0.14)$ matter sparing. Similarly, cavity volume was unchanged $(p=0.78$, unpaired

a
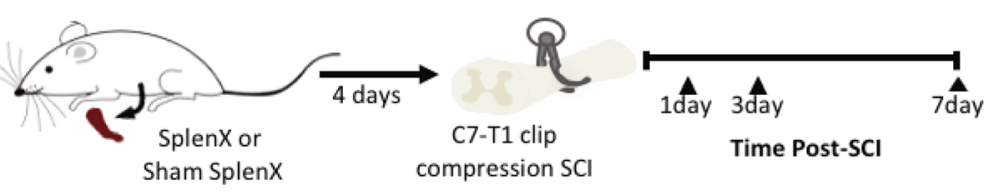

b

Parenchymal Hemorrhage
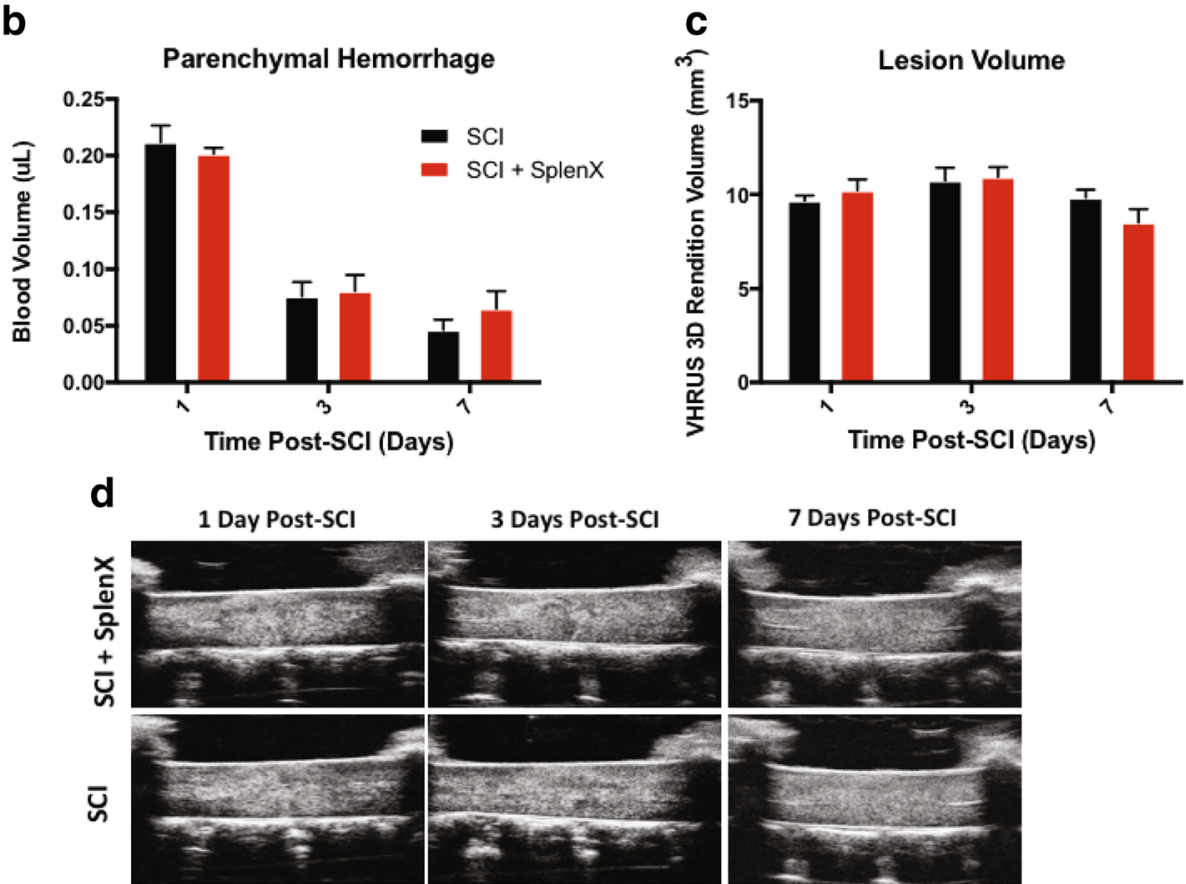

Fig. 2 Acute spinal cord parenchymal hemorrhage and lesion volume is unaffected by splenectomy at 1, 3, or 7 days following SCl. Schematic of experimental design for acute assessments (a). In short, splenectomies were performed 4 days prior to clip-compression C7-T1 SCI in Wistar rats. Drabkin's assay was applied to quantify parenchymal hemorrhage at 1 ( $n=5$ per group), 3 ( $n=7$ per group), and 7 ( $n=5$ per group) days post-SCl between the animals with or without a spleen (b). Very high-resolution ultrasound (VHRUS) imaging in B mode was also applied to quantify lesion volume (c). The representative sagittal view VHRUS images are shown at 1, 3, and 7 days post-SCI (d). Data are expressed as mean \pm SEM, and unpaired $t$ tests were performed at each time point with no significant differences found 


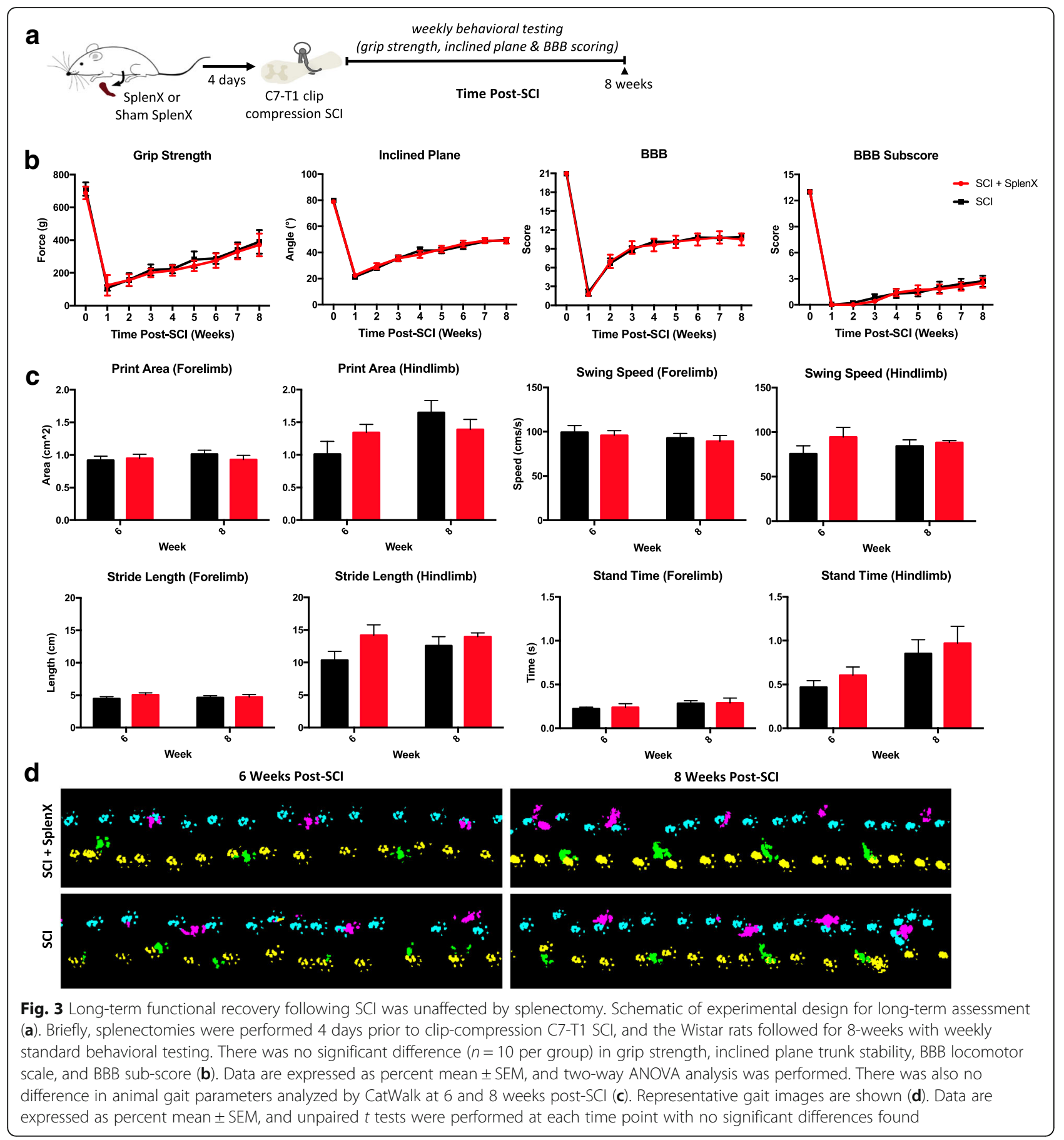

$t$ test) between splenectomised rodents $\left(1.05 \pm 0.13 \mathrm{~mm}^{3}\right)$ and those with a sham procedure $\left(1.01 \pm 0.06 \mathrm{~mm}^{3}\right)$.

Splenectomy alters the acute effects of early intravenous MSCs infusion following $\mathrm{SCl}$

Prior to in vivo use, HUCMCs (passages 7-8) were validated with positive expression of mesenchymal cell markers (CD73, CD146, CD44, CD13, and PDGFR) at 80-90\% confluence (Fig. 5b). Subsequently, as previously described [22],
$2.5 \times 10^{6}$ HUCMCs were infused via the tail vein at $1 \mathrm{~h}$ following SCI ( $n=4$ per group). The effects of cell infusion on splenic inflammation were evaluated at 1 day following SCI (Fig. 5c), where statistically significant $(p \leq .05)$ differences in cytokine expression levels were found for thymus chemokine/CXCL7 and sICAM (multiple $t$ tests with Holm-Sidak correction for multiple comparisons). Spinal cord lesion volume and systemic IL-10 expression were also evaluated at 1 day post-SCI and HUCMC infusion (Fig. 6a). Animals 


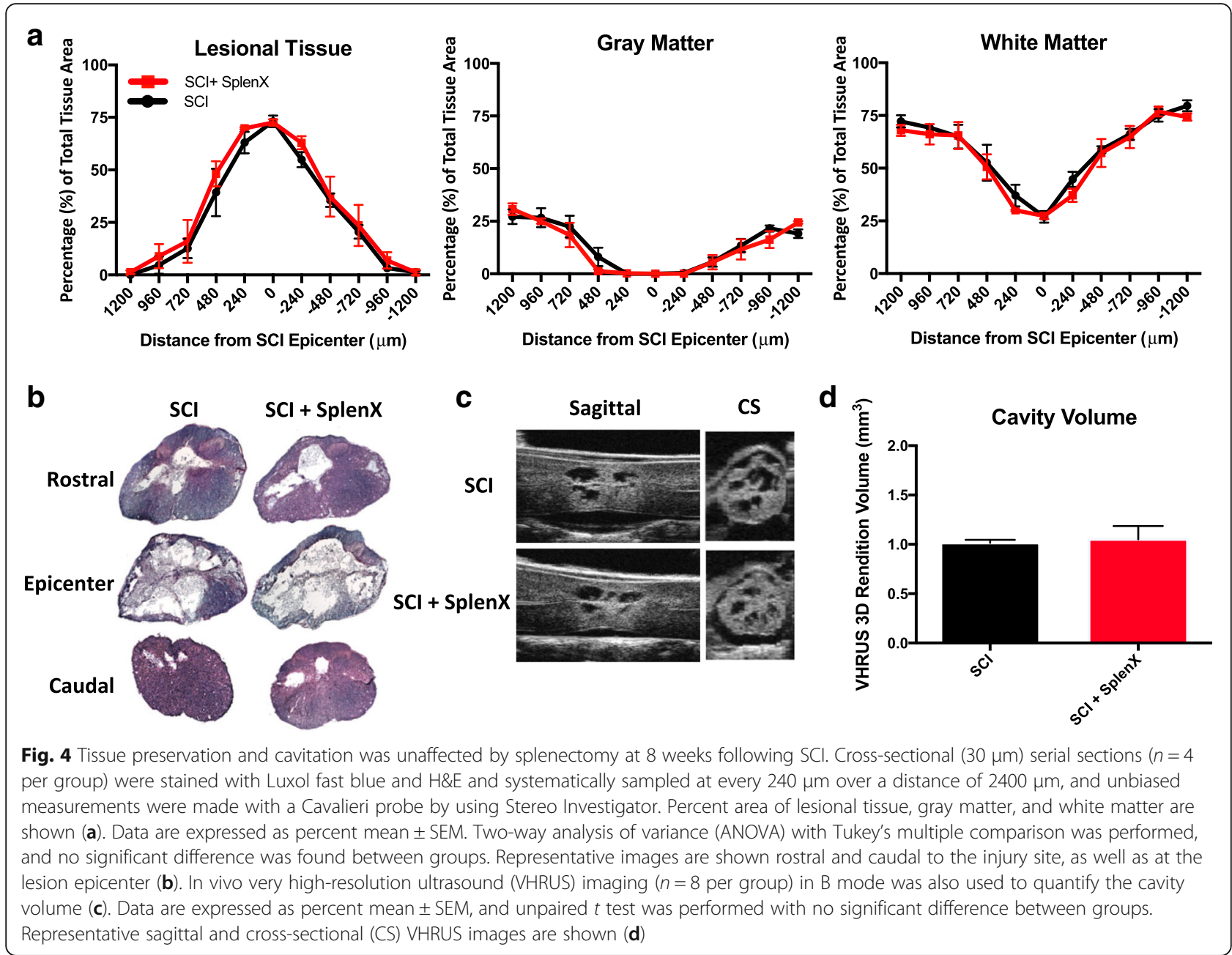

that received HUCMCs had a significantly ( $p=0.03$, unpaired $t$ test) reduced lesion volume $\left(7.96 \pm 0.93 \mathrm{~mm}^{3}\right)$ compared to that of vehicle controls $\left(11.89 \pm 0.60 \mathrm{~mm}^{3}\right)$. The concentration of plasma ( $p=0.009$, unpaired $t$ test $)$ and splenic IL-10 ( $p=0.03$, unpaired $t$ test) was also higher in HUCMC-treated animals. Interestingly, when cells were infused to splenectomised animals, some of the effects were lost (Fig. 6b). Specifically, the HUCMC-mediated reduction in VHRUS lesion volume was absent in splenectomized animals $(p=0.18$, unpaired $t$ test), despite a trend $(p=0.052$, unpaired $t$ test) in those with a sham procedure. Similarly, while there was less parenchymal hemorrhage with cells ( $p$ $=0.016$, unpaired $t$ test) in shams, this effect was lost in the splenectomy group ( $p=0.93$, unpaired $t$ test). The rise in plasma IL-10 levels was missing in both groups $(p=0.14$ for sham and $p=0.44$ for splenectomy, unpaired $t$ tests).

\section{Discussion}

Traumatic SCI drives local as well as systemic inflammation. Although the resulting peripheral pathology is known to contribute to multi-organ dysfunction, post-injury complications, and greater secondary damage to the spinal cord [30], the role of peripheral immune organs is poorly understood. For this reason, the therapeutic potential of lymphoid tissue targets in SCI remains largely unexplored. Here, we characterize the splenic inflammatory cytokine response to injury, demonstrating an early rise in TNFo levels that is followed by a significant influx of pro-inflammatory signaling (with increased expression of IL-17, IL-13, IL-1 $\beta$, and CXCL1). Despite previous reports [31], there was no significant difference in spleen weight or length in this injury model (Additional file 1: Figure S1). Relatedly, we further assessed the splenic involvement in secondary damage via splenectomy experiments. There was no significant difference in acute or long-term tissue damage, as well as functional recovery post-SCI, between splenectomised and sham surgery rodents. However, interestingly, when human umbilical cord matrix-derived MSCs (known as HUCMCs) were intravenously infused into splenectomised rodents, the previously reported protective effects [22] were lost. The splenic 


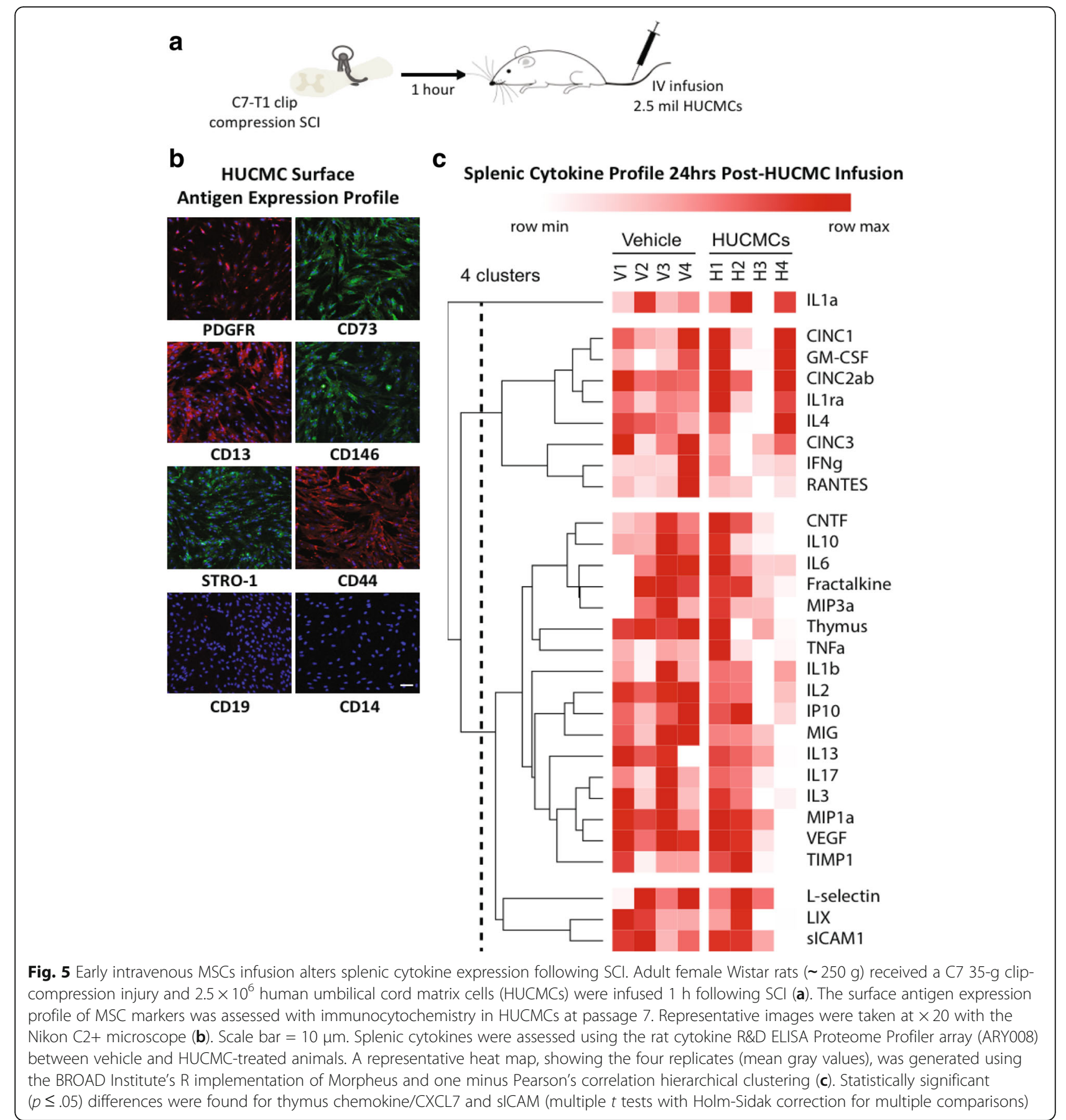

target of intravenous HUCMC infusion was further validated by demonstrating a significant reduction in inflammatory cytokines, thymus chemokine/CXCL7 and sICAM. Together, this work demonstrates that, while the inflammatory response function of the spleen can be replaced by other immune organs, it may be an important and clinically relevant therapeutic target for acute SCI pathology.

The rapid production of TNF $\alpha$ in the spleen post-trauma is consistent with reports in other models of injury [9], likely triggered by a combination of neural [32] as well as non-neural signaling [33], including angiotensin II. Considering TNFo's role as a mediator of inflammation [34], the subsequent rise in splenic pro-inflammatory molecules, albeit never previously reported, was expected. These results have further implications in the context of cell therapy, where TNF $\alpha$ has been reported to trigger MSC immunomodulatory effects [35-37], specifically through the production of TNFo-stimulated gene-6 (TSG6). It is 


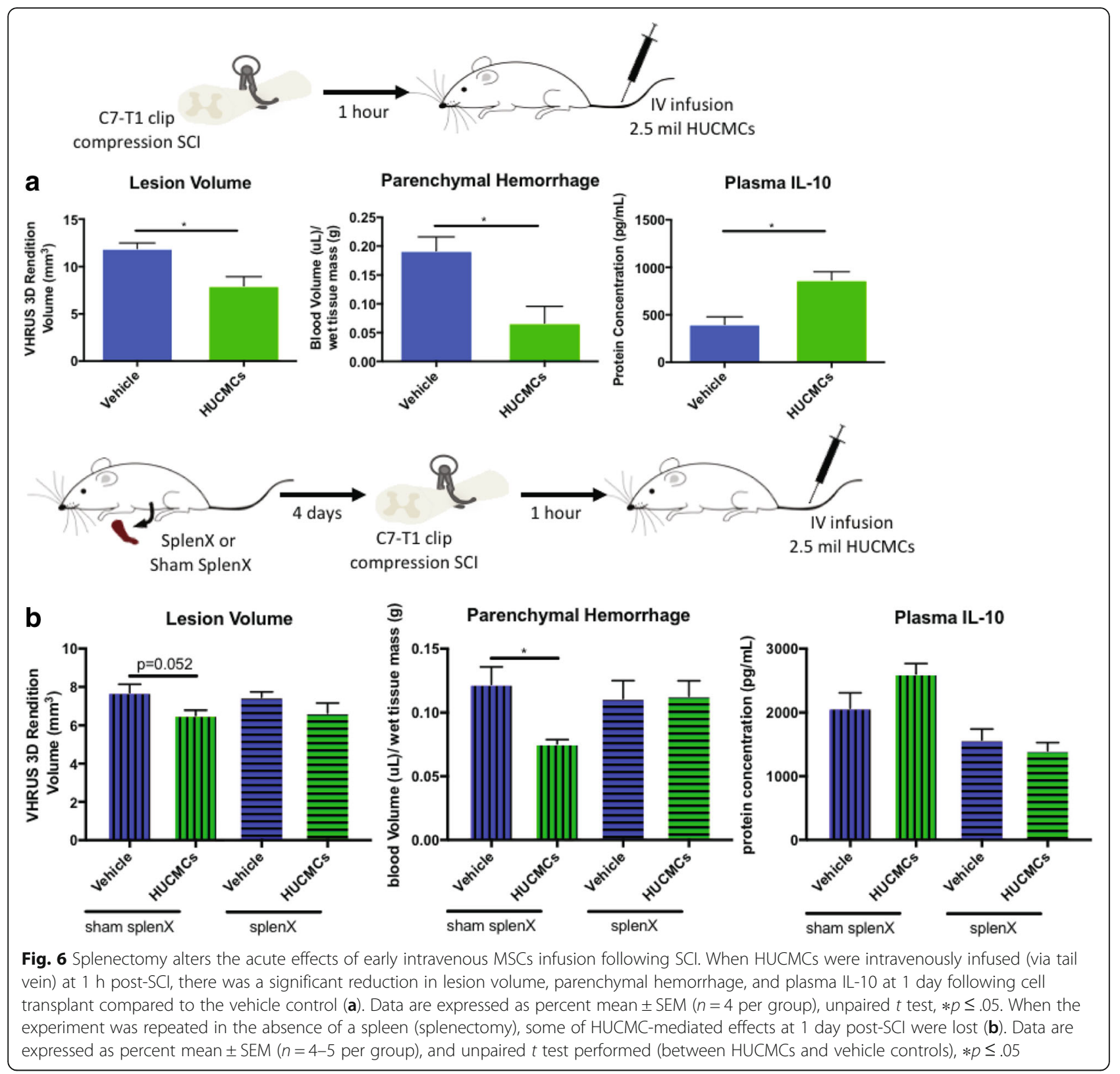

interesting to note that neonatal MSCs, specifically cells from the human umbilical cord, have been reported to exhibit enhanced and more rapid TSG6 expression with TNF $\alpha$ stimulation [38]. In turn, TSG6 has been shown to drive $\mathrm{M} \phi$ polarization to a more anti-inflammatory M2 state [39-41] and these cells are thought to contribute to recovery post-SCI [42, 43]. Other strategies reported to reprogram $M \phi s$ towards a more anti-inflammatory phenotype, such as peroxisome proliferator-activated receptor- $\gamma$ (PPAR- $\gamma$ ) [44], have also shown promise in SCI [45].

It is increasingly recognized that MSCs rarely localize to the site of injury and often mediate effects through short-lived engraftment in peripheral tissue [23]. Aside from the spleen [21], as described in our previous study [22], the lungs have also been widely reported as the tissue source of MSC-mediated effects post-SCI [46] in addition to other disease models [35]. Therefore, in the absence of a spleen, one would expect the lungs to play a central role in immunomodulation with MSCs. Yet, interestingly, reduced secondary pathology (as assessed by lesion volume and parenchymal hemorrhage) as well as the systemic rise in IL-10 following MSC infusion was lost in the splenectomy animals at acute time-points. If, as suggested by the profiling data, splenic TNF $\alpha$ production has an important role in activating transplanted MSCs, the absence may account for 
the changed outcomes. However, it is important to note that other MSC effects may endure and longer time-points are needed to further understand this relationship. Relatedly, previous work with human multipotent adult stem cells derived from bone marrow, known as multipotent adult progenitor cells (MAPC), demonstrated in stroke that intravenous cell effects on long-term functional recovery were lost with splenectomy [18]. However, there was also evidence of other MAPC targets, as the effects on lesion size were preserved without the spleen.

In contrast to the aforementioned studies, the bone marrow has been traditionally recognized as the primary source of circulating M $\phi s$ [47]. While precursors are found in the spleen [48], irradiation and splenectomy studies have demonstrated that the bone marrow alone can replenish circulating populations [47]. Therefore, with bone marrow able to replace some splenic function, the unchanged acute pathology and long-term recovery in splenectomised rodents can be explained by this compensation. Nevertheless, these results differ from the work of Blomster et al., who found that splenectomy improved outcomes in mouse SCI and identified the spleen as a key reservoir of $M \phi s$ responsible for exacerbating injury [14]. These differences may stem from species variation [49] as well as timing of splenectomy relative to trauma. Future work should aim to characterize the relationship between systemic, splenic, and bone marrow immune cell population as well as phenotype changes following trauma and MSC infusion.

With considerable blood influx and a large population of immune precursor cells, targeting the spleen for therapeutic purposes has numerous advantages. In one example, systemic delivery of nanoparticles encapsulating short interfering RNA (siRNA) against CCR2 allowed for silencing expression of this chemokine in splenic populations of inflammatory $M \phi s$, thereby attenuating their recruitment and tissue damage in various models of disease [50, 51]. Another study was also able to target the splenic $\mathrm{M} \phi$ progenitor recruitment by blocking angiotensin II, leading to improved outcomes in lung adenocarcinoma [52]. Together, these studies highlight the therapeutic potential of peripheral immune organ targets in neurotrauma, with the spleen as an exciting avenue for further exploration.

Herein, this work aimed to characterize a single mechanism of MSC-mediated action in the context of SCI. Although our assessment focused on the spleen, there are likely multiple mechanisms involved in the neuroprotective effects reported. For example, MSCs have been shown to coordinate the production of both matrix metalloproteinases and tissue inhibitors of matrix metalloproteinases (TIMPs) in order to mediate the structure of the vascular basement membrane and thereby vascular integrity [53]. The secretion of TIMPs (such as TIMP-3) by MSCs has been the suggested mechanism of intravenous cell delivery in a model of traumatic brain injury [19]. Other potential mechanisms include modulation of the VE-cadherin/ $\beta$-catenin signaling pathway [54] and secretion of Wnt3a [55] as well as prostaglandin E2 (PGE2) [56]. Moreover, this study has been largely limited to cytokine profiling, which only provides partial insight into physiological changes. Long-term assessment and further characterization of immune cell populations is warranted.

\section{Conclusions}

Overall, our study shows that the spleen, through amplification of inflammatory signaling, is involved in SCI secondary pathophysiology. Further, the spleen plays an important role in MSC-mediated immunomodulation, highlighting that peripheral immune tissues can be a therapeutic target for SCI. This finding can help us tailor cell therapy, as well as all systemic interventions, to maximize efficacy.

\section{Additional file}

Additional file 1: Figure S1. Spleen weight and length following traumatic $\mathrm{SCl}$. There was no significant difference in spleen weight (A) or length (B) at 1 or $24 \mathrm{~h}$ following SCI. Data are expressed as mean \pm SEM ( $n=4$ per group), one-way ANOVA with Tukey's multiple comparisons test. (PDF $354 \mathrm{~kb}$ )

\section{Abbreviations}

ANOVA: Analysis of variance; BBB: Basso, Beattie, and Bresnahan; ELISA: Enzymelinked immunosorbent assay; H\&E: Hematoxylin-eosin; LFB: Luxol fast blue; MAPC: Multipotent adult progenitor cells; MSCs: Mesenchymal stromal cells;

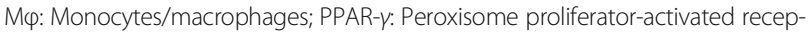
tor- $\gamma ;$ RIPA: Radioimmunoprecipitation assay; SCl: Spinal cord injury; SEM: Standard error of the mean; siRNA: Short interfering RNA; TNFa: Tumor necrotic factor-a; VHRUS: Very high-resolution ultrasound

\section{Acknowledgements}

The authors would like to acknowledge the Canadian Institutes of Health Research, the Government of Ontario, James F. Crothers Family, the Halbert Foundation, the Krembil Foundation, and the DeZwirek Family Foundation.

\section{Funding}

This work was supported by funds from the Canadian Institutes of Health Research Training Program in Regenerative Medicine (A.B.), the Ontario Graduate Scholarship (A.B.), and the James F. Crothers Family Fellowship in Peripheral Nerve Damage (A.B.). M.F. was supported by the Halbert Chair in Neural Repair and Regeneration, Krembil Foundation, and DeZwirek Family Foundation.

\section{Availability of data and materials}

The datasets used and/or analyzed during the current study are available from the corresponding author on reasonable request.

\section{Authors' contributions}

$A B$ made substantial contributions to the conception and design and acquisition, analysis, and interpretation of the data; was involved in drafting the manuscript and revising it critically for important intellectual content; gave the final approval of the version to be published; and agreed to be accountable for all aspects of the work in ensuring that questions related to the accuracy or integrity of any part of the work are appropriately investigated and resolved. JH made substantial contributions to the acquisition of the data and analysis, gave the final approval of the version to be published, and agreed to be accountable for all aspects of the work in ensuring that questions related to the accuracy or integrity of any part of the work are appropriately investigated and resolved. JH made substantial contributions to the acquisition of the data and analysis, gave the final 
approval of the version to be published, and agreed to be accountable for all aspects of the work in ensuring that questions related to the accuracy or integrity of any part of the work are appropriately investigated and resolved. MM made substantial contributions to the acquisition of the data and analysis, gave the final approval of the version to be published, and agreed to be accountable for all aspects of the work in ensuring that questions related to the accuracy or integrity of any part of the work are appropriately investigated and resolved. RV made substantial contributions to the acquisition of the data and analysis, gave the final approval of the version to be published, and agreed to be accountable for all aspects of the work in ensuring that questions related to the accuracy or integrity of any part of the work are appropriately investigated and resolved. MGF made substantial contributions to the conception and design, was involved in drafting the manuscript or revising it critically for important intellectual content, gave the final approval of the version to be published, and agreed to be accountable for all aspects of the work in ensuring that questions related to the accuracy or integrity of any part of the work are appropriately investigated and resolved.

\section{Ethics approva}

All animal experiments were approved by the animal care committee at the University Health Network (Toronto, Ontario, Canada) in compliance with the Canadian Council on Animal Care.

\section{Consent for publication}

Not applicable.

\section{Competing interests}

The authors declare that they have no competing interests.

\section{Publisher's Note}

Springer Nature remains neutral with regard to jurisdictional claims in published maps and institutional affiliations.

\section{Author details}

'Division of Genetics and Development, Krembil Research Institute, University Health Network, 60 Leonard Ave, Toronto, Ontario M5T 2S8, Canada. ${ }^{2}$ Institute of Medical Science, University of Toronto, 1 King's College Circle, Toronto, Ontario M5S 1A8, Canada. ${ }^{3}$ Division of Neurosurgery, Toronto Western Hospital, 399 Bathurst St. Suite 4WW-449, Toronto, Ontario M5T 2S8, Canada.

Received: 16 February 2018 Accepted: 28 June 2018 Published online: 03 August 2018

\section{References}

1. Ulndreaj A, Badner A, Fehlings MG. Promising neuroprotective strategies for traumatic spinal cord injury with a focus on the differential effects among anatomical levels of injury. F1000Res. 2017;6:1907.

2. Tator $\mathrm{CH}$, Fehlings MG. Review of the secondary injury theory of acute spinal cord trauma with emphasis on vascular mechanisms. J Neurosurg. 1991;75:15-26

3. Anthony DC, Couch Y. The systemic response to CNS injury. Exp Neurol. 2014;258:105-11.

4. Jung W-C, Levesque J-P, Ruitenberg MJ. It takes nerve to fight back: the significance of neural innervation of the bone marrow and spleen for immune function. Semin Cell Dev Biol. 2016.

5. Lucin KM, Sanders VM, Jones TB, Malarkey WB, Popovich PG. Impaired antibody synthesis after spinal cord injury is level dependent and is due to sympathetic nervous system dysregulation. Exp Neurol. 2007;207:75-84.

6. Brommer B, Engel O, Kopp MA, Watzlawick R, Müller S, Prüss H, et al. Spinal cord injury-induced immune deficiency syndrome enhances infection susceptibility dependent on lesion level. Brain J Neurol. 2016;139:692-707.

7. Ueno M, Ueno-Nakamura Y, Niehaus J, Popovich PG, Yoshida Y. Silencing spinal interneurons inhibits immune suppressive autonomic reflexes caused by spinal cord injury. Nat Neurosci. 2016:19:784-7.

8. Ulndreaj A, Tzekou A, Mothe AJ, Siddiqui AM, Dragas R, Tator CH, et al. Characterization of the antibody response after cervical spinal cord injury. J Neurotrauma. 2017;34:1209-26.
9. Swirski FK, Nahrendorf M, Etzrodt M, Wildgruber M, Cortez-Retamozo V, Panizzi $P$, et al. Identification of splenic reservoir monocytes and their deployment to inflammatory sites. Science. 2009;325:612-6.

10. Ajmo CT, Vernon DOL, Collier L, Hall AA, Garbuzova-Davis S, Willing A, et al. The spleen contributes to stroke-induced neurodegeneration. J Neurosc Res. 2008:86:2227-34.

11. Bao Y, Kim E, Bhosle S, Mehta H, Cho S. A role for spleen monocytes in postischemic brain inflammation and injury. J Neuroinflammation. 2010;7:92.

12. Seifert HA, Hall AA, Chapman CB, Collier LA, Willing AE, Pennypacker KR. A transient decrease in spleen size following stroke corresponds to splenocyte release into systemic circulation. J Neuroimmune Pharmacol Off J Soc Neurolmmune Pharmacol. 2012;7:1017-24.

13. Zhang Y, Guan Z, Reader B, Shawler T, Mandrekar-Colucci S, Huang K, et al. Autonomic dysreflexia causes chronic immune suppression after spinal cord injury. J Neurosci. 2013:33:12970-81.

14. Blomster LV, Brennan FH, Lao HW, Harle DW, Harvey AR, Ruitenberg MJ. Mobilisation of the splenic monocyte reservoir and peripheral $C_{3}$ CR1 deficiency adversely affects recovery from spinal cord injury. Exp Neurol. 2013;247:226-40.

15. Kim E, Yang J, Beltran CD, Cho S. Role of spleen-derived monocytes/ macrophages in acute ischemic brain injury. J Cereb Blood Flow Metab Off Int Soc Cereb Blood Flow Metab. 2014;34:1411-9.

16. Zierath D, Shen A, Stults A, Olmstead T, Becker KJ. Splenectomy does not improve long-term outcome after stroke. Stroke. 2017:48:497-500.

17. Acosta SA, Tajiri N, Hoover J, Kaneko Y, Borlongan CV. Intravenous bone marrow stem cell grafts preferentially migrate to spleen and abrogate chronic inflammation in stroke. Stroke. 2015;46:2616-27.

18. Yang B, Hamilton JA, Valenzuela KS, Bogaerts A, Aronowski J, Mays RW, et al Multipotent adult progenitor cells enhance recovery after stroke by modulating the immune response from the spleen. Stem Cells Dayt Ohio. 2017:35:1290-302

19. Menge T, Zhao Y, Zhao J, Wataha K, Gerber M, Zhang J, et al. Mesenchymal stem cells regulate blood-brain barrier integrity through TIMP3 release after traumatic brain injury. Sci Transl Med. 2012;4:161ra150.

20. Walker PA, Shah SK, Jimenez F, Gerber MH, Xue H, Cutrone R, et al. Intravenous multipotent adult progenitor cell therapy for traumatic brain injury: preserving the blood brain barrier via an interaction with splenocytes. Exp Neurol. 2010;225:341-52.

21. DePaul MA, Palmer M, Lang BT, Cutrone $R$, Tran AP, Madalena KM, et al. Intravenous multipotent adult progenitor cell treatment decreases inflammation leading to functional recovery following spinal cord injury. Sci Rep. 2015;5:16795.

22. Badner A, Vawda R, Laliberte A, Hong J, Mikhail M, Jose A, et al. Early intravenous delivery of human brain stromal cells modulates systemic inflammation and leads to vasoprotection in traumatic spinal cord injury. Stem Cells Transl Med. 2016:5:991-1003.

23. Fabian C, Naaldijk Y, Leovsky C, Johnson AA, Rudolph L, Jaeger C, et al. Distribution pattern following systemic mesenchymal stem cell injection depends on the age of the recipient and neuronal health. Stem Cell Res Ther. 2017:8:85.

24. Soubeyrand M, Badner A, Vawda R, Chung YS, Fehlings MG. Very high resolution ultrasound imaging for real-time quantitative visualization of vascular disruption after spinal cord injury. J Neurotrauma. 2014;31:1767-75.

25. Choudhri TF, Hoh BL, Solomon RA, Connolly ES, Pinsky DJ. Use of a spectrophotometric hemoglobin assay to objectively quantify intracerebra hemorrhage in mice. Stroke. 1997:28:2296-302.

26. Forgione N, Karadimas SK, Foltz WD, Satkunendrarajah K, Lip A, Fehlings MG. Bilateral contusion-compression model of incomplete traumatic cervical spinal cord injury. J Neurotrauma. 2014;31:1776-88.

27. Rivlin AS, Tator $\mathrm{CH}$. Objective clinical assessment of motor function after experimental spinal cord injury in the rat. J Neurosurg. 1977;47:577-81.

28. Basso DM, Beattie MS, Bresnahan JC. A sensitive and reliable locomotor rating scale for open field testing in rats. J Neurotrauma. 1995;12:1-21.

29. Nguyen DH, Cho N, Satkunendrarajah K, Austin JW, Wang J, Fehlings MG. Immunoglobulin $\mathrm{G}$ (lgG) attenuates neuroinflammation and improves neurobehavioral recovery after cervical spinal cord injury. J Neuroinflammation. 2012:9:224.

30. Sun X, Jones ZB, Chen X-M, Zhou L, So K-F, Ren Y. Multiple organ dysfunction and systemic inflammation after spinal cord injury: a complex relationship. J Neuroinflammation. 2016;13:260

31. Lankford KL, Arroyo EJ, Nazimek K, Bryniarski K, Askenase PW, Kocsis JD. Intravenously delivered mesenchymal stem cell-derived exosomes target M2-type macrophages in the injured spinal cord. PLoS One. 2018;13:e0190358. 
32. Seeley EJ, Barry SS, Narala S, Matthay MA, Wolters PJ. Noradrenergic neurons regulate monocyte trafficking and mortality during gram-negative peritonitis in mice. J Immunol Baltim Md 1950. 2013;190:4717-24.

33. Cortez-Retamozo V, Etzrodt M, Newton A, Rauch PJ, Chudnovskiy A, Berger C, et al. Origins of tumor-associated macrophages and neutrophils. Proc Natl Acad Sci U S A. 2012;109:2491-6.

34. Baud V, Karin M. Signal transduction by tumor necrosis factor and its relatives. Trends Cell Biol. 2001;11:372-7.

35. Lee RH, Pulin AA, Seo MJ, Kota DJ, Ylostalo J, Larson BL, et al. Intravenous hMSCs improve myocardial infarction in mice because cells embolized in lung are activated to secrete the anti-inflammatory protein TSG-6. Cell Stem Cell. 2009;5:54-63.

36. Choi H, Lee RH, Bazhanov N, Oh JY, Prockop DJ. Anti-inflammatory protein TSG-6 secreted by activated MSCs attenuates zymosan-induced mouse peritonitis by decreasing TLR2/NF-KB signaling in resident macrophages. Blood. 2011;118:330-8

37. Lee RH, Yu JM, Foskett AM, Peltier G, Reneau JC, Bazhanov N, et al. TSG-6 as a biomarker to predict efficacy of human mesenchymal stem/progenitor cells (hMSCs) in modulating sterile inflammation in vivo. Proc Natl Acad Sci U S A. 2014;111:16766-71.

38. Hamidian Jahromi S, Li Y, Davies JE. Effect of Tumor Necrosis Factor Alpha Dose and Exposure Time on Tumor Necrosis Factor-Induced Gene-6 Activation by Neonatal and Adult Mesenchymal Stromal Cells. Stem Cells Dev. 2018;27(1):44-54. https://doi.org/10.1089/scd.2017.0179.

39. Song W-J, Li Q, Ryu M-O, Ahn J-O, Ha Bhang D, Chan Jung Y, et al. TSG-6 secreted by human adipose tissue-derived mesenchymal stem cells ameliorates DSS-induced colitis by inducing M2 macrophage polarization in mice. Sci Rep. 2017;7:5187.

40. Mittal M, Tiruppathi C, Nepal S, Zhao Y-Y, Grzych D, Soni D, et al. TNFastimulated gene-6 (TSG6) activates macrophage phenotype transition to prevent inflammatory lung injury. Proc Natl Acad Sci U S A. 2016;113: E8151-8.

41. Martinez FO, Gordon S. The M1 and M2 paradigm of macrophage activation: time for reassessment. F1000prime Rep. 2014;6:13.

42. Shechter R, London A, Varol C, Raposo C, Cusimano M, Yovel G, et al. Infiltrating blood-derived macrophages are vital cells playing an antiinflammatory role in recovery from spinal cord injury in mice. PLoS Med. 2009;6:e1000113

43. Kigerl KA, Gensel JC, Ankeny DP, Alexander JK, Donnelly DJ, Popovich PG. Identification of two distinct macrophage subsets with divergent effects causing either neurotoxicity or regeneration in the injured mouse spinal cord. J Neurosci. 2009;29:13435-44.

44. Ren $Y$, Young W. Managing inflammation after spinal cord injury through manipulation of macrophage function. Neural Plast. 2013; 2013:945034.

45. McTigue DM, Tripathi R, Wei P, Lash AT. The PPAR gamma agonist Pioglitazone improves anatomical and locomotor recovery after rodent spinal cord injury. Exp Neurol. 2007;205:396-406.

46. White SV, Czisch CE, Han MH, Plant CD, Harvey AR, Plant GW. Intravenous transplantation of mesenchymal progenitors distribute solely to the lungs and improve outcomes in cervical spinal cord injury. Stem Cells Dayt Ohio. 2016;34:1812-25

47. van Furth $\mathrm{R}$, Cohn ZA. The origin and kinetics of mononuclear phagocytes J Exp Med. 1968;128:415-35.

48. Geissmann F, Manz MG, Jung S, Sieweke MH, Merad M, Ley K. Development of monocytes, macrophages, and dendritic cells. Science. 2010;327:656-61.

49. Bronte $\mathrm{V}$, Pittet MJ. The spleen in local and systemic regulation of immunity. Immunity. 2013;39:806-18.

50. Leuschner F, Dutta P, Gorbatov R, Novobrantseva TI, Donahoe JS, Courties $G$, et al. Therapeutic siRNA silencing in inflammatory monocytes in mice. Nat Biotechnol. 2011;29:1005-10.

51. Leavy O. Immunotherapy: stopping monocytes in their tracks. Nat Rev Immunol. 2011;11:715.

52. Cortez-Retamozo V, Etzrodt M, Newton A, Ryan R, Pucci F, Sio SW, et al. Angiotensin II drives the production of tumor-promoting macrophages. Immunity. 2013;38:296-308

53. Lozito TP, Kuo CK, Taboas JM, Tuan RS. Human mesenchymal stem cells express vascular cell phenotypes upon interaction with endothelial cell matrix. J Cell Biochem. 2009;107:714-22.

54. Menge T, Gerber M, Wataha K, Reid W, Guha S, Cox CS, et al. Human mesenchymal stem cells inhibit endothelial proliferation and angiogenesis via cell-cell contact through modulation of the VE-cadherin/ $\beta$-catenin signaling pathway. Stem Cells Dev. 2013;22: 148-57.

55. Zhao Y, Gibb SL, Zhao J, Moore AN, Hylin MJ, Menge T, et al. Wnt3a, a protein secreted by mesenchymal stem cells is neuroprotective and promotes neurocognitive recovery following traumatic brain injury. Stem Cells Dayt Ohio. 2016;34:1263-72.

56. Vasandan AB, Jahnavi S, Shashank C, Prasad P, Kumar A, Prasanna SJ. Human mesenchymal stem cells program macrophage plasticity by altering their metabolic status via a PGE2-dependent mechanism. Sci Rep. 2016;6:38308.

\section{Ready to submit your research? Choose BMC and benefit from:}

- fast, convenient online submission

- thorough peer review by experienced researchers in your field

- rapid publication on acceptance

- support for research data, including large and complex data types

- gold Open Access which fosters wider collaboration and increased citations

- maximum visibility for your research: over $100 \mathrm{M}$ website views per year

At BMC, research is always in progress.

Learn more biomedcentral.com/submissions 